\title{
Carcinoma of gallbladder: Clinical evaluation and survival rate at a tertiary care center in Rajasthan
}

\author{
Gopal Goyal $^{1}$ - Kumar Shwetanshu Narayan ${ }^{1}$ - Gaurav Kumar Gupta ${ }^{1}$. \\ Sandeep Nijhawan ${ }^{1}$
}

Published online: 12 July 2017

(C) Indian Society of Gastroenterology 2017

Sir,

Gallbladder cancer (GBC) is one of the most common gastrointestinal (GI) malignancies seen in India and fifth most common malignancy of the GI tract [1]. The distribution of GBC is geographically heterogeneous with the highest incidence rate (up to 21.5 per 100,000 population) being observed in India. Although many large case series from the Indian subcontinent have explored the etiological and clinical aspect of GBC, there are very few published case series of therapeutic interventions and subsequent follow up. We describe the clinical picture, natural course, and the result of therapeutic interventions in a prospectively followed cohort of GBC patients presenting to our tertiary health care center.

A total of 140 consecutive patients of GBC from October 2013 to March 2015 were enrolled in the study. The mean (SD) age of patients was 53 (12.7) years and female:male ratio was 2.41:1. Pain abdomen, jaundice, and GB lump were present in $78.5 \%, 68.5 \%$, and $24.2 \%$, respectively. Gallstones were present in $52.1 \%$. Out of 140 patients, $42(30 \%)$ had advanced disease and so were offered the best supportive care. Thirty patients $(21.4 \%)$ had resectable disease on the basis of imaging and therefore underwent exploratory laparotomy. At the time of laparotomy, 15 turned out to be unresectable. In the remaining 15 patients, 3 had stage II disease and underwent radical cholecystectomy while 12 patients had stage III disease and underwent extended surgical resection. Sixty- eight of 140 patients had unresectable disease and were offered palliative treatment in the form of stenting, percutaneous biliary drainage, and

Sandeep Nijhawan

dr_nijhawan@yahoo.com

1 Department of Gastroenterology, Sawai Man Singh Medical College, J.L.N. Marg, Jaipur 302 004, India surgical bypass. Overall, surgery was carried out in $30(21 \%)$ patients and endoscopic treatment in $44(31 \%)$ patients and no interventions were carried out in the remaining patients because of disseminated disease. On follow up, survival rate for stage II, stage IIIa, stage IIIb, stage IVa, and stage IVb was $>12,10$, $8.75,4.5$, and 1.5 months, respectively.

The present study highlights that carcinoma of the gallbladder predominantly affected females $(70.7 \%)$ in middle age. The constellation of clinical features and etiological profile of GBC patients was similar to that reported earlier from India and other countries [2, 3]. The median survival of GBC patients was dismal in the present study as majority of them presented in advanced stage, similar to previous studies from different parts of India [2, 4]. We found that endoscopic ultrasound plays an important role in diagnosing mass lesion in GB neck which is difficult to pick up otherwise. Additionally, we found that contrast-enhanced CT scans understage the GBC disease, as half of the patients who were resectable on CT turned out to be unresectable on laparotomy.

\section{References}

1. Dhir V, Mohandas KM. Epidemiology of digestive tract cancers in India. IV. Gall bladder and pancreas. Indian $\mathrm{J}$ Gastroenterol. 1999;18:24-8.

2. Batra Y, Pal S, Dutta U, et al. Gallbladder cancer in India: a dismal picture. J Gastroenterol Hepatol. 2005;20:309-14.

3. Shukla VK, Khandelwal C, Roy SK, Vaidya MP. Primary carcinoma of the gallbladder: a review of a 16 year period at the university hospital. J Surg Oncol. 1985;28:32-5.

4. Pandey M, Pathak AK, Gautam A, Aryya NC, Shukla VK. Carcinoma of the gallbladder: a retrospective review of 99 cases. Dig Dis Sci. 2001;46:1145-51. 